\title{
Resistência à mefloquina do tipo RIII em crianças com malária falciparum em Manaus, AM, Brasil
}

\author{
RIII mefloquine resistance in children with falciparum \\ malaria in Manaus, AM, Brazil \\ Elza Noronha, Maria das Graças Alecrim, Gustavo Adolfo \\ Sierra Romero e Vanize Macêdo
}

\begin{abstract}
Resumo Relata-se a ocorrência de resistência à mefloquina administrada na dose de $20 \mathrm{mg} / \mathrm{kg}$ em 51 crianças com malária falciparum atendidas em centro de referência em Manaus, Brasil,, no período de outubro a novembro de 1997. Todas as crianças foram avaliadas nos dias 3, 5, 7, 14, 21, 28 e 35 do tratamento, segundo critérios clínico e parasitológico. Foi encontrada uma incidência de resistência RIII de 5,9\% (IC 95\% variando de 1,5 a 17,2), a razão cura/resistência calculada foi 20:1 e cura/gravidade 62:1. Os dados chamam a atenção para a importância da resistência à mefloquina nesse grupo de crianças.
\end{abstract}

Palavras-chaves: Malária. P. falciparum. Mefloquina. Resistência. Crianças.

Abstract We report the occurrence of resistance to mefloquine 20mg/day in 51 children with falciparum malaria treated, at reference center of Manaus, Brazil, from October to December 1997. All children were evaluated at day 3, 5, 7, 14, 21, 28 and 35 of treatment. Clinical and parasitological cure criteria were adopted. The incidence of RIII mefloquine resistance was 5.9\% (IC 95\% 1.5-17.2). The cure/resistance proportion was 20:1 and cure/severity was 62:1. These findings suggest the importance of mefloquine resistance within this group of children.

Key-works: Malaria. P. falciparum. Mefloquine. Resistance. Children.

A resistência aos antimaláricos tem ampla distribuição no mundo e é um dos entraves para o controle da malária, valendo lembrar que a disseminação de cepas de $P$. falciparum resistentes à cloroquina, hoje, praticamente se sobrepõe à distribuição geográfica da endemia. A resistência do plasmódio às drogas está relacionada a fatores ligados ao parasita, ao hospedeiro e às condições sócio-econômicas e, geralmente, o uso frequente, abusivo e indiscriminado dos medicamentos corrobora para a disseminação de cepas resistentes.

Na Amazônia Brasileira, a multirresistência do P. falciparum foi verificada desde a década de
$80^{126}$, fazendo-se necessária a busca de medicações alternativas mais eficazes, como a mefloquina. Apesar de alguns estudos de sensibilidade in vivo ${ }^{313}$ mostrarem resistência $\mathrm{RI}$ do Plasmodium falciparum à mefloquina nessa região, a caracterização in vitro, geralmente, aponta para a boa resposta à droga ${ }^{918}$. Em contrapartida, em um estudo longitudinal realizado no Estado do Pará ${ }^{7}$, foi observada a tendência ao aumento das concentrações inibitórias mínimas da mefloquina para o plasmódio ao longo do tempo, sugerindo a possibilidade de diminuição da sensibilidade com o uso da droga a longo prazo. De uma maneira geral, até o presente momento,

Núcleo de Medicina Tropical e Nutrição da Universidade de Brasília, Brasília, DF e Instituto de Medicina Tropical do Amazonas, Manaus, AM, Brasil.

Endereço para correspondência: Dra Elza Noronha. Núcleo de Medicina Tropical e Nutrição/UNB. Caixa Postal 4517, 70919-970 Brasília, DF, Brasil.

Tel: 55 61 273-5008/348-2197, Fax: 5561 273-2811

e-mail: tropical@unb.br

Recebido para publicação em 25/5/99. 
não existem dados suficientes relacionados à resistência que impossibilitem o uso da mefloquina para o tratamento da malária falciparum no Brasil.

A problemática da resistência às drogas antimaláricas torna-se ainda maior quando se considera a infecção pelo $P$. falciparum em crianças, por se tratar de um grupo etário onde a doença está potencialmente relacionada com maior morbidade e mortalidade. Relata-se nesse trabalho, a ocorrência de resistência à mefloquina, com padrão RIII, em crianças tratadas em centro de referência em Manaus, AM, Brasil.

\section{MATERIAL E MÉTODOS}

Durante um estudo clínico, realizado no Instituto de Medicina Tropical do Amazonas (IMTAM), na cidade de Manaus, no período de outubro a dezembro de 1997, foram acompanhadas 51 crianças com idade de até 14 anos, com diagnóstico de malária falciparum não complicada, tratadas com mefloquina na dose de $20 \mathrm{mg} / \mathrm{kg}$ fracionada em duas doses com intervalo de 6 horas. Garantiu-se que os pacientes não tivessem história de convulsões ${ }^{17}$ e todos foram avaliados segundo os critérios de gravidade estabelecidos pela Organização Mundial de Saúde (OMS) ${ }^{19}$. Cada criança teve uma ficha clínica contendo dados de anamnese e clínicos. O diagnóstico laboratorial da malária foi realizado por meio de gota espessa com coloração pelo Giemsa ${ }^{4}$, sendo realizada a contagem de parasitos em relação a 100 leucócitos com posterior cálculo da densidade parasitária. A primeira dose da mefloquina foi administrada sob supervisão e a segunda, em casa pelos pais ou agentes de cuidados, sendo orientados para a possibilidade de intercorrências. O controle de cura foi feito nos dias 3, 5, 7,14, 21, 28 e 35, sendo registrado o desaparecimento da febre e da parasitemia assexuada. O padrão de sensibilidade in vivo foi avaliado segundo a OMS ${ }^{11}$. Os pacientes resistentes foram tratados com associação de artemether em duas doses: $2 \mathrm{mg} / \mathrm{kg} / \mathrm{dia}$ e $1 \mathrm{mg} / \mathrm{kg} / \mathrm{dia}$.

O protocolo de pesquisa foi submetido e aprovado pela Comissão de Ética do IMT-AM e cumpriu com as exigências da Resolução 196/96 do Conselho Nacional de Saúde sobre Desenvolvimento de Pesquisa em Seres Humanos. A análise de dados foi realizada no Programa EPI-INFO versão 6.04. A freqüência da resistência foi estimada por meio de proporção com seu respectivo Intervalo de Confiança de $95 \%$ com nível de significância de 5\% e os eventos incidentes foram expressos pela relação pacientes/dias de observação.

\section{RESULTADOS}

Foram acompanhadas 51 crianças com média de idade de 7,5 anos (desvio padrão de 4,2 anos), sendo semelhantes as proporções entre os sexos: $45,1 \%$ para o feminino e $54,9 \%$ para o masculino. Houve perdas amostrais no seguimento dos pacientes visto que, dos 51 que iniciaram o estudo, somente 36 compareceram para verificação de cura no terceiro dia, 10 no quinto, 29 no sétimo, 15 no $14^{\circ}, 13$ no $21^{\circ} \stackrel{\circ}{ }, 11$ no $28^{\circ}$ e 4 no $35^{\circ}$ dia. Uma criança foi excluída da análise de resistência pois apresentou aumento da parasitemia (de 3825 para 43775 parasitas $/ \mathrm{mm}^{3}$ ) associado a crise convulsiva, sendo necessário o uso de artemether no segundo dia de tratamento.

Os principais sintomas e sinais encontrados nos pacientes, previamente ao tratamento, estão mostrados na Tabela 1. No exame físico, 98\% das crianças não apresentavam alterações do estado geral. Entre os achados anormais, 49\% tinham hepatomegalia, 43,1\% esplenomegalia,
33,3\% palidez cutâneo-mucosa e 5,9\% desidratação leve.

Em relação à resposta terapêutica, três (5,9\%) pacientes (IC $95 \%$ de 1,5 a 17,2 ) apresentaram malária resistente à mefloquina. O padrão de resistência foi caracterizado como RIII, como está representado na Figura 1, onde se observa um gráfico logarítimico, mostrando a tendência do comportamento das parasitemias. Todas as três crianças tinham relato de episódio anterior de diarréia e duas de vômitos. Dessas, uma evolui para gravidade, tendo apresentado hiperparasitemia, infecção pulmonar e hemoglobinúria. Em todas as crianças, houve negativação da parasitemia até o sétimo dia de tratamento, após o uso de artemether. Não foram referidos outros efeitos adversos nas demais crianças.

$\mathrm{Na}$ tentativa de aproveitamento dos dados, foi utilizada a variável pacientes/tempo para 
estabelecer as relações entre alguns eventos incidentes nos primeiros sete dias pós tratamento. Os índices de cura, resistência e de gravidade calculados para o grupo de crianças foram os seguintes: 373 casos/1.000 pacientes/dia, 18 casos/1.000 pacientes/dia e 6 casos $/ 1000$ pacientes dia, permitindo estabelecer uma relação de cura/resistência de 20:1 e cura/gravidade de 62:1.

Tabela1- Principais manifestações clínicas em 51 crianças com malária falciparum tratadas com mefloquina no IMT-AM, Brasil.

\begin{tabular}{lcccc}
\hline Sintomas e sinais & № & $\%$ & Duração* $^{*}$ & Desvio padrão \\
\hline Febre & 50 & 98,0 & 4,8 & 4,7 \\
Cefaléia & 41 & 80,4 & 4,4 & 2,8 \\
Calafrios & 35 & 68,6 & 4,2 & 3,0 \\
Sudorese & 33 & 64,7 & 4,2 & 2,8 \\
Mialgia & 30 & 58,8 & 4,1 & 2,8 \\
Náuseas & 28 & 54,9 & 5,0 & 5,7 \\
Lombalgia & 26 & 51,0 & 4,0 & 2,9 \\
Vômitos & 24 & 47,1 & 4,3 & 3,1 \\
Tosse & 24 & 47,1 & 5,5 & 6,2 \\
Artralgia & 17 & 33,3 & 3,8 & 3,2 \\
Diarréia & 15 & 29,4 & 4,0 & 3,0 \\
Dispnéia & 5 & 9,8 & 3,8 & 0,9 \\
Tontura & 3 & 5,9 & 4,0 & 1,0
\end{tabular}

* média de dias

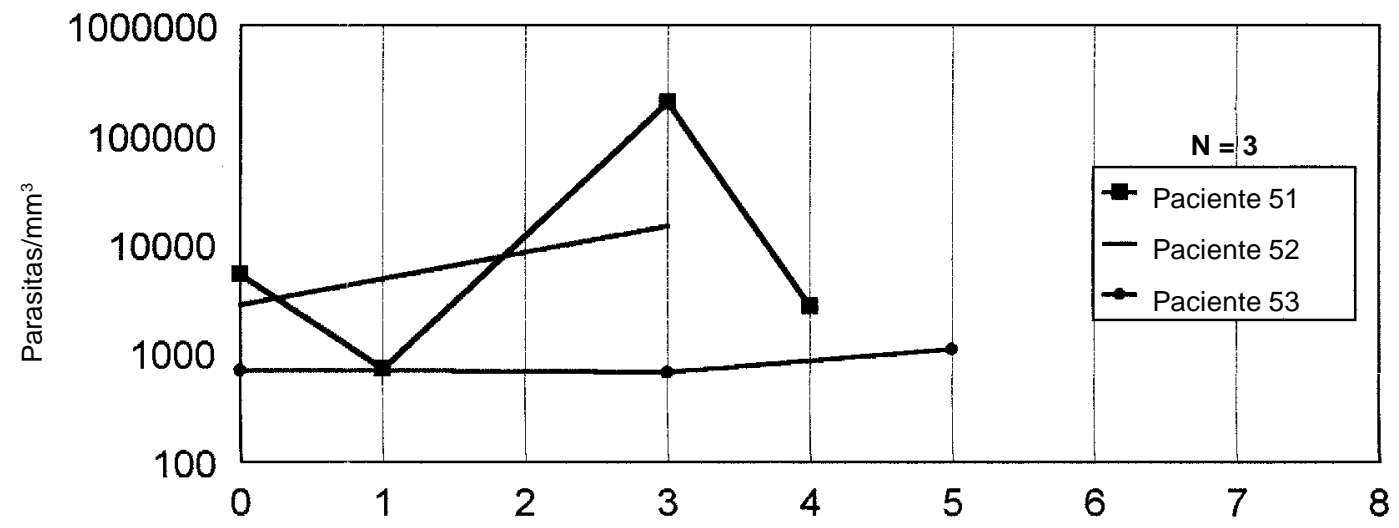

\begin{tabular}{|l|c|c|c|r|r|r|r|c|}
\multicolumn{1}{c|}{} & \multicolumn{10}{c|}{ dias de acompanhamento } \\
\cline { 2 - 9 } \multicolumn{1}{c|}{} & $\mathbf{0}$ & $\mathbf{1}$ & $\mathbf{2}$ & $\mathbf{3}$ & $\mathbf{4}$ & $\mathbf{5}$ & $\mathbf{6}$ & $\mathbf{7}$ \\
\hline Paciente 51 & $\mathbf{5 2 5 2}$ & $\mathbf{7 0 7}$ & NR & $\mathbf{2 0 0 9 0 0}$ & $\mathbf{2 6 2 6}$ & $\mathbf{0}$ & $\mathbf{0}$ & $\mathbf{0}$ \\
Paciente 52 & $\mathbf{2 7 4 5}$ & NR & NR & $\mathbf{1 4 4 9 0}$ & $\mathbf{0}$ & NR & NR & NR \\
Paciente 53 & $\mathbf{6 9 3}$ & NR & NR & $\mathbf{6 6 0}$ & NR & $\mathbf{1 0 5 6}$ & $\mathbf{0}$ & $\mathbf{0}$ \\
\hline
\end{tabular}

$\mathrm{NR}=$ não realizado

Figura1 - Padrão RIII de resistência em três pacientes com malária falciparum tratados com mefloquina, no IMTAM, Brasil. 


\section{DISCUSSÃO}

A mefloquina foi introduzida no Brasil como alternativa terapêutica para malária falciparum multirresistente na década de 80 , quando foram realizados os primeiros ensaios clínicos de eficácia ${ }^{12} 141516$ e tem sido usada, com relativa segurança, no tratamento das crianças com malária falciparum ${ }^{10}$ e em associação com derivados da artemisinina, na malária grave e nas cepas de $P$. falciparum resistentes às múltiplas drogas 5 .

O achado de resistência RIII à mefloquina encontrado nesse estudo aponta para a necessidade de melhor monitorização da sensibilidade da droga nas crianças tratadas no IMT-AM e para a importância do fenômeno na determinação da maior morbidade pela doença, fazendo-se necessário estudos mais aprofundados como a caracterização de fatores de risco para a resistência, visando o uso racional da medicação.
Alguns desses fatores foram estudados em crianças na Tailândia ${ }^{8}$, sendo verificado que a baixa idade, a presença de diarréia nos primeiros dois dias do tratamento, a alta parasitemia, a anemia e tratamentos anteriores estavam relacionados com o insucesso terapêutico. No presente estudo não foi possível estabelecer esse tipo de correlação, embora se tenha observado a ocorrência de diarréia e vômitos previamente ao tratamento, o que poderia ter influenciado a absorção da droga, interferindo com a boa resposta clínica.

Relatamos, pela primeira vez, a ocorrência de resistência RIII à mefloquina em crianças da Amazônia, o que poderá contribuir para a reavaliação da terapêutica utilizada correntemente no IMT-AM, em Manaus, sugerindo a necessidade de uso mais racional da mefloquina, bem como de padronização de drogas alternativas mais eficazes e seguras.

\section{AGRADECIMENTOS}

Às crianças e aos seus pais pela colaboração, ao Prof. Wilson Duarte Alecrim pelas facilidades oferecidas para realização desse estudo no
IMT-AM, ao Sr. Pedro S. Lopes e à Sra. Raimunda Barreto pela revisão do diagnóstico parasitológico.

\section{REFERÊNCIAS BIBLIOGRÁFICAS}

1. Alecrim MGC. Estudo da resistência do $P$. falciparum às drogas antimaláricas in vivo e in vitro na Amazônia. Tese de Mestrado, Universidade de Brasília, Brasília, DF, 1981.

2. Alecrim MGC. Resistance to in vivo and in vitro chemotherapies in the Brazilian Amazonia. Memórias do Instituto Oswaldo Cruz 81:153-157, 1986.

3. Alecrim MGC, Alecrim WD, Albuquerque BC, Silva EB. Fracasso no tratamento da malária ( $P$. falciparum) com novas drogas antimaláricas In: Resumos do XXII Congresso da Sociedade Brasileira de Medicina Tropical, p. 91, 1986.

4. Antuñano FJL. Diagnóstico microscópico de los párasitos de la malaria en la sangre In: Antuñano FJL, Schmunis G (eds) Diagnóstico de malaria. Organización Panamericana de la Salud, Washington, p.39-50, 1988.

5. Bunnag D, Kanda T, Karbwang J, Thimasarn K, Pungpak $\mathrm{S}$, Harisanuta T. Artemether or artesunate followed by mefloquine as a possible treatment for multidrug resistant falciparum malaria. Transactions of the Royal Society of Tropical Medicine and Hygiene 90:415-417, 1996.

6. Couto AA, Calvosa VS, Santos MA, Souza JM. Resistência in vitro de cepas do $P$. falciparum isoladas no sul do Estado do Pará, diferentes períodos: emergência de casos de multirresistência. Revista da Sociedade Brasileira de Medicina Tropical 26:5-9, 1993.

7. Couto AA, Calvosa VS, Santos MA, Souza JM. Evolução temporal da resistência in vitro do $P$. falciparum às drogas antimaláricas em duas áreas da Amazônia Brasileira com distintas características sócio-econômicas e geográficas. Revista da Sociedade Brasileira de Medicina Tropical 28:357-365, 1995.

8. Fontanet AL, Walker AM. Predictors of treatment failure in multiple drug-resistant falciparuma malaria: results from 42-day follow-up of 224 patients in Eastern Thailand. The American Journal of Tropical Medicine and Hygiene 49:465-472, 1993.

9. Neifer S, Kremsner PG. Drug susceptibility of Plasmodium falciparum in the western amazon region, State of Acre, Brazil. Revista do Instituto de Medicina Tropical de São Paulo 33:205-211, 1991.

10. Okoyeh JN, Lege-Oguntoye L, Ugbode RO, Ogunrinde GO. Responses of multidrug-resistant Plasmodium falciparum parasites to mefloquine in Nigerian children. Tropical Medicine and International Health 2:319-324, 1997.

11. Organizácion Mundial de la Salud. Quimioterapia del paludismo. Serie de informes tecnicos, Genebra, $n^{\circ} \mathbf{3 7 5}$, 1967. 
12. Santos JB, Prata A. Tratamento de 75 pacientes portadores de malária pelo $P$. falciparum, em Porto Velho (RO), com mefloquina. Revista da Sociedade Brasileira de Medicina Tropical 21:181-185, 1988.

13. Silva AR, Rebelo MM, Mendes W. Malária II. Resposta do Plasmodium falciparum ao tratamento no Estado do Maranhão In: Resumo do XXI Congresso da Sociedade Brasileira de Medicina Tropical, p. 158, 1995.

14. Souza JM. A phase I clinical trial of mefloquine in Brazilian male subjects. Bulletin of the World Health Organization 61:815-820, 1983.

15. Souza JM. A phase II clinical trial of mefloquine in Brazilian male subjects. Bulletin of the World Health Organization 61:809-814, 1983.

16. Souza JM, Sheth UK, Oliveira RMG, Roulet H, Souza SD. An open, randomized, phase III clinical trial of mefloquine and of quinine plus sulfadoxinepyrimethamine in the treatment of symptomatic falciparum malaria in Brazil. Bulletin of the World Health Organization 63:603-609, 1985.

17. Sowonmi A, Alako LA, Oduola AMJ, Walker O, Akindele JA, Ogundahunsi OAT. Neuropsychiatric side effects of mefloquine in Africans. Transactions of the Royal Society of Tropical Medicine and Hygiene 87:462-463,1993.

18. Zalis MG, Pang L, Silveira MS, Milhous WK, Wirth DF. Characterization of Plasmodium falciparum isolated from the Amazon region of Brazil: Evidence for quinine resistance. American Journal of Tropical Medicine and Hygiene 58:630-637, 1998.

19. Warrel DA, Molineaux ME, Beales PF. Severe and complicated malaria. Transactions of the Royal Society of Tropical Medicine and Hygiene 84:1-65, 1990. 